\title{
Author Correction: Ex situ seed banks and the IUCN Red List
}

Sarah E. Dalrymple and Thomas Abeli

Correction to: Nature Plants https://doi.org/10.1038/s41477-019-0366-3, published online 08 February 2019.

In the version of this Comment originally published, the authors mistakenly omitted an Acknowledgements section, which should have read: "This research was partly supported by grants from the Grant of Excellence Departments, MIUR, Italy (grant nos. ARTICOLO 1, COMMI 314-337 and LEGGE 232/2016 to T.A.)". This has now been amended. 